\title{
Mikrobiologische Diagnostik der periprothetischen Infektion
}

\author{
Lars Frommelt
}

\section{Zusammenfassung}

Die periprothetische Infektion ist eine fremdkörperassoziierte Infektion, die sich im umgebenden Knochengewebe manifestiert und in den meisten Fällen aus einer intraoperativen bakteriellen Kontamination resultiert. Diese Infektion manifestiert sich meist erst nach Monaten bis Jahren und wird deswegen häufig nicht mit der zugrundeliegenden Operation in Verbindung gebracht. Dazu kommt, dass diese Infektionen häufig sehr symptomarm sind und leicht mit einer aseptischen Lockerung verwechselt werden können. Die Anzahl der Erreger ist in diesen Fällen meist gering, was bei der Diagnostik berücksichtigt werden muss. Die Kenntnis der Pathogenese gestattet es dem Operateur, das diagnostische Vorgehen und die Therapie zu planen. In diesem Zusammenhang ist die Fähigkeit der Erreger, Biofilm zu bilden, von grundlegender Bedeutung und erklärt, warum die Entfernung des implantierten Fremdmaterials (Kunstgelenke genauso wie Osteosynthesematerial oder vertebrale Stabilisierungen) notwendig ist, um diese Infektion zu beherrschen. Diese Arbeit ist darauf ausgerichtet, grundlegende Informationen zum Verständnis von fremdkörperassoziierten Infektionen zu vermitteln, um die Diagnostik im klinischen Alltag zu verbessern.

\section{Microbiological Diagnosis of Periprosthetic Infection}

Periprosthetic infection is a foreign body-associated infection that is characterised by delayed osteomyelitis of the bone tissue surrounding an artificial joint element. Most of these infections result from intraoperative bacterial contamination of the prosthetic device that is implanted. The period from contamination to clinically apparent infection often amounts to months or even years. In these cases the initial surgical procedure is frequently not considered as causative factor for the actual infection. Moreover, these infections often produce minimal symptoms that can be mistaken for aseptic loosening of the artificial joint apparatus. The bacterial burden of the causative pathogen is low in such cases and this has to be considered in the diagnostic procedures. Knowledge of the pathogenesis also allows the orthopaedic surgeon to plan the diagnostic procedures and therapy. In this context the ability of the pathogen to form a biofilm has a crucial impact and explains why removal of the foreign body is necessary to control this type of infection. This paper is intended to provide some basic information for a better understanding of foreign body-associated infection in order to improve the practice of diagnostics in clinical routine.

\section{Einleitung}

Die periprothetische Infektion ist eine der gefürchteten Komplikationen in der Gelenkersatzchirurgie. Sie ist mit einer

OP-JOURNAL 2010; 26: 32-36

(C) Georg Thieme Verlag KG Stuttgart · New York DOI http://dx.doi.org/10.1055/s-0030-1250185

Fällen kann die Diagnose durch eine qualifizierte mikrobiologische Diagnostik gesichert werden.

Diese Diagnostik ist ein übergreifender Prozess, der in der Klinik beginnt, im Labor fortgesetzt wird und nach gemeinsamer Evaluierung der Ergebnisse durch die beteiligten Fachdisziplinen die weitere Behandlung beeinflusst. Die fachübergreifende Kommunikation ist hier, ähnlich wie in der Onkologie, erforderlich, um die Behandlungsergebnisse zu verbessern.

Ziel dieser Arbeit ist es, die mikrobiologische Diagnostik als fachübergreifenden Prozess darzustellen und Voraussetzungen, die sich aus der Pathogenese dieser Erkrankung ergeben, darzustellen.

\section{Definition und Pathogenese der periprothetischen Infektion}

Die periprothetische Infektion ist eine fremdkörperassoziierte Infektion, die in der Umgebung eines dauerhaft in den Körper eingebrachten Fremdmaterials durch Bakterien hervorgerufen wird.

Diese Infektion nimmt in den meisten Fällen ihren Ausgang von einer intraoperativen Kontamination der Prothese, die zu einer Besiedelung des Fremdkörpers führt. Es folgt dann eine langsame Ausbreitung der Bakterien entlang der Fremdkörperoberfläche im Interface zwischen Fremdmaterial und Knochengewebe.

Es kommt dabei zu einer Veränderung der Eigenschaften der Bakterien. Die Bakterien gelangen in die OP-Wunde in der stoffwechselaktiven planktonischen Form und gehen bei Kontamination der Fremdkörperoberfläche unter Bildung von Biofilm in die sessile, stoffwechselinaktive Form über. Dieser Übergang wird durch Botenstoffe (Pheromone) ermöglicht, die die vorhandenen Bakterien dazu bringen, synchron Biofilm zu bilden
Frequenz von ca. $1 \%$ bezogen auf Erstir von Kunstgelenken ein se und im klinischen Ersch gsbild nicht eindeutig und leicht mit einer aseptischen Lockerung eines Kunstgelenks erwechselt werden. Durch den zeitlichen Verlauf wird eine Verbindung zu der Implantation der Prothese nicht hergestellt. In den meisten 
und ihren Stoffwechsel zu reduzieren. Diese Kommunikation der Bakterien untereinander wird Quorum sensing genannt. Im Gegensatz zu planktonischen sind sessile Bakterien gegen Antibiotika weitgehend unempfindlich und durch den Biofilm vor der Elimination durch phagozytierende Zellen geschützt. Die periprothetische Infektion manifestiert sich, wenn ein Teil der sessilen Bakterien wiederum in die planktonische Form übergeht und in dem umgebenden Gewebe eine Infektionskrankheit, die periprothetische Infektion hervorruft. Es handelt sich also um eine Osteomyelitis in der Umgebung des Fremdkörpers.

Die periprothetische Infektion ist eine Osteomyelitis des umgebenden Knochengewebes bei Kunstgelenken, die von sessilen Bakterien im Biofilm auf der Prothesenoberfläche als Keimreservoir unterhalten wird. Sessile Bakterien können weder durch die Abwehr noch durch Antibiotika eliminiert werden.

Auch wenn hämatogene Infektionen vorkommen und die Ausbreitung der Erreger per continuitatem möglich ist, gelangt der überwiegende Teil der Erreger bei Implantation eines Kunstgelenks aus der Hautflora an die Oberfläche der Prothese (intraoperative Kontamination). Begünstigend ist dabei, dass die Menge der Erreger, die notwendig ist, diesen Prozess in Gang zu bringen, extrem niedrig ist. Unabhängig davon, wie die Bakterien an die Prothese gelangen, folgt der Kontamination die Kolonisation und der Übergang in die sessile Form im Biofilm. Im Biofilm breiten sich die Bakterien entlang der Fremdkörperoberfläche aus. Dieser Vorgang kann Monate bis Jahre dauern, bis die periprothetische Infektion manifest wird.

Die Infektion selbst wird von planktonischen Bakterien unterhalten; die sessilen Bakterien im Biofilm auf der Prothese dienen dabei als Reservoir, sodass eine Infektberuhigung der periprothetischen Osteomyelitis durch Antibiotika nur scheinbar zur Kontrolle der Infektion führt. Deswegen ist zur Sanierung dieser Infektion die chirurgische Entfernung der Prothese, abgesehen von Infektionen im frühen Verlauf, erforderlich.

\section{Mikrobiologische Diagnostik der periprothetischen Infektion - Klinischer Part: Probengewinnung und -management}

Die Diagnose einer fremdkörperassoziierten Infektion ist häufig schwierig, da die klinische Symptomatik nicht in allen Fällen eindeutig ist und voraussetzt, dass dem behandelnden Arzt das Krankheitsbild geläufig ist. Schmerzen sind das führende Symptom. Bei periprothetischen Infektionen von Knie- und Hüftgelenken sind lokale Symptome wie Rötung, Schwellung, Überwärmung oder eine Fistel nur in ca. einem Drittel der Fälle vorhanden.

Am Anfang der Diagnostik steht der Verdacht, der sich aus dem klinischen Erscheinungsbild, ggf. einer radiologisch $\mathrm{zu}$ vermutenden Lockerung und Laborwerten wie einer erhöhten Blutsenkungsgeschwindigkeit sowie einem erhöhten C-reaktiven Protein ergibt. Beweisend ist der Erregernachweis.

\section{Probenart und -gewinnung}

Der Goldstandard für die Diagnostik von Knochen- und Gelenkinfektionen ist die Knochen- bzw. Synovialisbiopsie mit mikrobiologischer und histologischer Untersuchung.

Wird eine periprothetische Infektion eines Kunstgelenks vermutet, ist eine mikrobiologische und zytologische Untersuchung der Synovialflüssigkeit sinnvoll und führt in den meisten Fällen zur Diagnose.

\section{Gelenkpunktion}

Die Gelenkpunktion ist anders als bei anderen fremdkörperassoziierten Infektionen in der Orthopädie eine geeignete Untersuchungsmethode, da durch die Ausbreitung der Bakterien im Biofilm entlang der Implantatoberfläche in den meisten Fällen bei Manifestation der periprothetischen Infektion der Gelenkraum erreicht wurde und die Bakterien dann in der Synovialflüssigkeit z.T. auch als planktonische Formen vorliegen, die deutlich besser anzuzüchten sind.

Ein weiterer Vorteil von Gelenkpunktionen besteht darin, dass die bei Mikroskopie sichtbare zelluläre Reaktion im Gelenkraum einen zusätzlichen Hinweis auf die Wahrscheinlichkeit einer periprothetischen Infektion liefert.
Gewinnung der Probe Qualität der Entnahme sichert die Qualität des Erregernachweises

Da die Erregerzahl in der Regel, abgesehen von Gelenkempyemen, gering ist, muss bei Gewinnung der Probe auf eine ausreichende Probenmenge (mindestens $1 \mathrm{ml}$ ) geachtet werden.

Die Probe muss unter streng aseptischen Bedingungen mit chirurgischer Hautdesinfektion, steriler Abdeckung und sterilem Kittel, Haube und Mund-/Nasenschutz für den Punkteur in einem Eingriffsraum oder Operationsraum gewonnen werden, wie dies den Vorgaben der Richtlinie für Krankenhaushygiene und Infektionsprävention des Robert KochInstituts in Berlin für Punktionen von sterilen Körperhöhlen vorgegeben ist. Die Intention der Hygienerichtlinie ist zwar der Patientenschutz, aber das beschriebene Vorgehen stellt auch sicher, dass die Probe so kontaminationsfrei wie möglich gewonnen wird. Vor dem Hintergrund, dass die Erreger der periprothetischen Infektion sich aus der Hautflora rekrutieren, ist die Vermeidung von Kontaminationen wichtig. Auch die Erreger der Infektion stammen überwiegend aus der Hautflora und weisen insbesondere bei symptomarmen Infektionen nur geringe Keimzahlen auf, sodass eine Abgrenzung von Erregern und Kontaminanten in einigen Fällen nicht möglich ist.

Wegen der meist geringen Anzahl der Erreger in den Proben ist eine primäre Verdünnung der Proben durch „Anspülen“ nicht sinnvoll, da auf diese Weise die Erreger unter die Nachweisgrenze der klassischen mikrobiologischen Diagnostik verdünnt werden können.

Lokalanästhetika besitzen in vitro einen bakteriostatischen Effekt. Die Auswirkungen auf die Proben werden kontrovers diskutiert und es fehlen belastbare Studien dazu. Von daher sollte eine direkte Kontamination der Proben mit Lokalanästhetika vermieden werden.

Punktionen unter laufender Antibiotikatherapie haben eine hochgradig eingeschränkte Aussagekraft. Antibiotika sollten mindestens 2 Wochen vor Gewinnung der Proben abgesetzt werden. Dies ist bei der periprothetischen Infektion als chronische Infektion in aller Regel ohne Gefährdung des Patienten möglich [9]. Bei Patienten mit septikämischen Verlaufsformen verbietet sich diese Vor- 
gehen und eine schnelle chirurgische Revision ist erforderlich. In diesen Fällen sollten Blutkulturen für den Erregernachweis versucht werden. Da bestimmte Erreger wie Streptokokken nach Antibiotikaexposition häufig kulturell nicht mehr anzüchtbar sind, sollte in diesen Fällen auch eine molekularbiologische Untersuchung (s.u.) erwogen werden.

Da für diese Infektionen auch empfindliche Erreger, wie z.B. Anaerobier, in Betracht kommen, sollten die Proben zügig (innerhalb weniger Stunden) in das untersuchende Labor verbracht werden. Auch ein Transport in Transportmedien gewährleistet nicht das sichere Überleben und die Anzüchtbarkeit von empfindlichen Erregern. Sollte bei Punktaten absehbar sein, dass eine Verarbeitung der Proben nicht zeitgerecht möglich ist, besteht bei Punktaten die Möglichkeit, diese auf Blutkulturflaschen zu verimpfen und damit eine primäre Kultur anzulegen. Hier sind sog. pädiatrische Blutkultursysteme von Vorteil, da diese weitgehend unabhängig von der Menge des Inokulums sind.

Die Entnahmebedingungen bestimmen die Qualität der Probe:

- Entnahme der Probe unter streng aseptischen Kautelen (OP-artige Bedingungen $=$ Vermeidung von bakterieller Kontamination)

- Probenverdünnung z. B. durch „Anspülen" erschwert den Erregernachweis

- ausreichende Probenmenge erforderlich (Minimum $1 \mathrm{ml}$ )

- direkte Exposition der Probe mit Lokalanästhetika kann den Erregernachweis erschweren

- Antibiotikatherapie 14 Tage vor Probengewinnung beenden (Ausnahme: septikämische Patienten!)

Die Vorgehensweise sollte jeweils mit dem versorgenden mikrobiologischen Labor abgestimmt werden.

\section{Punktat: Zytologische Untersuchung}

Bei Punktaten sollte in jedem Fall eine zytologische Untersuchung durchgeführt werden, die mit einer Wahrscheinlichkeit von 0,8 mit einer periprothetischen Infektion korreliert, wenn bei künstlichen Kniegelenken die Zellzahl $1700 \mu \mathrm{l}$ überschreitet, und 65\% neutrophile Granulozyten nachweisbar sind [9].

Der Autor hat bei semiquantitativen Auswertungen von Gelenkpunktaten keinen Unterschied zwischen Gelenkpunktaten aus Hüft- und Kniegelenken gefunden.
In jeden Fall ist die präoperative zytologische Untersuchung ein wertvoller Hinweis auf eine periprothetische Infektion. Hierzu wird ein Aliquot der Gelenkflüssigkeit in ein mit ethylenediaminetetone acid (EDTA) präpariertes Röhrchen überführt, wie es für hämatologische Routineuntersuchungen benutzt wird. Dabei ist auf die korrekte Füllung der Röhrchen zu achten, und diese Diagnostik muss im Vorfeld mit dem untersuchenden Labor abgestimmt werden, da diese Proben mit Hyaluronidase versetzt werden müssen, um ein Verklumpen der Synovialflüssigkeit zu verhindern. Damit ist die Untersuchung auf Hämatologie-Analysern möglich.

\section{Biopsien}

Die Knochenbiopsie oder die Biopsie der periprothetischen Membran sind die am besten geeigneten Proben für die mikrobiologische und histologische Untersuchung. Diese Proben werden typischerweise während eines Revisionseingriffs gewonnen und stehen damit in der Regel präoperativ nicht zur Verfügung.

\section{Intraoperative Biopsien}

Bei chronischen Infektionen ist mit nur geringen Keimzahlen zu rechnen, sodass bei Probengewinnung für die mikrobiologische Untersuchung ausreichend große Proben (mindestens erbsengroß) gewonnen werden sollten.

Die zytologische Analyse der Synovialflüssigkeit gibt Hinweise auf das Vorliegen einer periprothetischen Infektion unabhängig vom Ergebnis der mikrobiologischen Kultur.

Da diese Proben unter Sicht gewonnen werden, können die Biopsien auf verdächtigen Arealen gewonnen werden, und es besteht auf diese Weise bei der periprothetischen Infektion eine hohe Treffsicherheit in Bezug auf den Erreger.

Bei Biopsien sollten mehrere Biopsien (mindestens 5) untersucht werden, da es auf diese Weise möglich ist, durch Nachweis identischer Mikroorganismen aus mehreren Biopsien die Spezifität des Erregernachweises in Abgrenzung zu Kontaminaten auf $98 \%$ zu steigern [1].

\section{Präoperative Biopsien}

Fink und Mitarbeiter [2] konnten zeigen, dass präoperative Biopsien gezogen auf den intraoperativen Erregernachweis die Gesamtrichtigkeit (Accuracy) der Ergebnisse von $89 \%$ bei Gelenkpunktionen auf $98 \%$ steigern konnten. Vorteil dieser Methode ist, dass wie bei den Biopsien allgemein schon beschrieben, Kontaminationen schon präoperativ eliminiert werden können.

\section{Transport von Biopsien}

Der Transport der Biopsien sollte in sterilen Gefäßen erfolgen, in die 1-2 Tropfen steriler 0,9\%iger Kochsalz vorgelegt werden, um eine Austrocknen auf dem Transport zu verhindern. Auch hier sollten die Transportzeiten gering gehalten werden.

Ist ein zeitgerechter Transport nicht möglich, ist ein Transport im anaeroben Milieu (Anaerobiertopf oder Plastik-Bag mit Gasgeneratoren) möglich.

\section{Sonikation von Prothesen}

Der Goldstandard für die mikrobiologische Untersuchung ist bislang die Untersuchung von Knochenbiopsien oder Biopsien aus der periprothetischen Membran. Bessere Ergebnisse können erzielt werden, wenn das Fremdmaterial einer Sonikation zugeführt wird, wobei die im Biofilm adhärenten Bakterien mittels Ultraschall aus dem Biofilm befreit werden und großer Zahl zur Untersuchung zur Verfügung stehen. Trampuz [10] beschreibt eine deutliche Verbesserung der Ausbeute. Diese Art der Untersuchung setzt allerdings voraus, dass die Prothese vollständig zur Untersuchung zur Verfügung steht.

Zurzeit steht diese Art der Untersuchung nur an wenigen Stellen zur Verfügung und ist mit großen logistischen Problemen behaftet.

Die Sonikation der gesamten Prothese sichert den Erregernachweis und die Abgrenzung von bakteriellen Kontaminationen.

\section{Ungeeignete Proben}

Abstriche, die nur einen Teil der zur Verfügung stehenden Keime transportieren können, sind ungeeignet, da eine Reduktion der Erreger in der Methode begründet ist und damit falsch negative Ergebnisse vorprogrammiert sind [3].

Fistelabstriche zeigen fast immer Wachstum von Bakterien. Die Abgrenzung der Erreger von sekundärer, bakterieller Besiedelung der Fistel ist schwer und in 
vielen Fällen nicht möglich. Die Diskussion zu diesem Proben wird kontrovers geführt; übereinstimmend wird nur der alleinige bzw. der vorherrschende Nachweis von Staphylococcus aureus ätiologisch als richtungsweisend angesehen. Die Ergebnisse von Fistelabstrichen sind häufig nicht zu interpretieren, und die Ergebnisse sind nur mit größter Zurückhaltung zu verwerten [6].

- Abstriche sind ungeeignete Proben!

- Fistelabstriche sind zum Erregernachweis ungeeignet und aufgrund der bakteriellen Sekundärbesiedelung verwirrend.

\section{Mikrobiologische Diagnostik der peri- prothetischen Infektion - Analytischer Part: Probenverarbeitung im Labor}

Für die mikrobiologische Untersuchung im Labor ist es entscheidend, das dem Labor mitgeteilt wird, dass eine periprothetische Infektion vermutet wird, da für diese Proben aufgrund der veränderten Eigenschaften der Erreger spezielle Untersuchungsgänge erforderlich sind.

\section{Klassische Mikrobiologie}

Welche Bedeutung die klinischen Informationen haben, wird klar, wenn man sich die Ergebnisse der Gelenkpunktionen bezogen auf die Biopsie der intraoperativen Infektmembran vergegenwärtigt. Der präoperative Erregernachweis gelingt nach eigener Erfahrung in einer Gesamtrichtigkeit (Accuracy) von $92 \%$ bei Sensitivität von $82 \%$ und einer Spezifität von 96\% [2]. Vor dem Hintergrund der Literatur variiert die Accuracy von $90-100 \%$, die Sensitivität von $12-$ $100 \%$ und die Spezifität von $81-100 \%$. Die größte Varianz besteht hinsichtlich der Sensitivität. Als Ursache sind die unterschiedlichen bakteriologischen Methodiken in den Studien zu vermuten.

Bezüglich der mikrobiologischen Verarbeitung sollte diese entsprechend den Mikrobiologisch-infektiologischen Qualitätsstandards (MIQ) 18 und 19 [4] erfolgen. Hier sind auch Regeln für die Probengewinnung hinterlegt. Eine Beobachtungszeit von 2 Wochen ist bei fremdkörperassoziierten Infektionen empfohlen und erhöht die Rate der nachgewiesenen Erreger deutlich [7].

Fink und Mitarbeiter zeigten in einer Studie, dass sich durch die Kombination von Gelenkpunktion und 5 Biopsien eine Accuracy von $98,6 \%$ (positiver prädikti- ver Wert [PPV]: 95,2\%; negativer prädiktiver Wert [NPV]: 100\%) durch synoptische Betrachtung von mikrobiologischen Befunde aus Punktat und Biopsien erreichen lässt [2].

Die Histologie ist sehr geeignet, spezifische Läsionen wie tuberkulöse Granulome aufzuzeigen. Bezüglich der Frage periprothetische Infektion wurde eine Accuracy von 93,8\% (PPV: 87,8\%; NPV: $96,1 \%)$ erreicht.

\section{Molekularbiologische Mikrobiologie}

Die molekularbiologischen Untersuchungsmethoden, die auf der Amplifizierung von erregerspezifischer Erbsubstanz (Nukleinsäure-Amplifikationstest - NAT) beruhen, sind für den Erregernachweis für die Diagnostik der Osteomyelitiden bislang nicht hinreichend evaluiert.

In der Diagnostik spezieller Erreger wie Mykobakterien, Borrelien, Bartonella henselae u.a. ist es häufig die einzige Methode, um zu einem zeitgerechten Erregernachweis zu kommen.

Mittlerweile sind Multiplex-NATs verfügbar, die in der Lage sind, einen Großteil der möglichen Erreger, insbesondere auch der Koagulase-negativen Staphylokokken („Staphylococcus-epidermidisGruppe“) zu detektieren, und die damit ergänzend zur klassischen Mikrobiologie bei der Diagnostik der Erreger hilfreich sind [5]. Nachteil dieser Methoden ist, dass zzt. über die Resistenz gegenüber Antibiotika nur sehr eingeschränkte Aussagen getroffen werden können. Bei der zunehmenden Multiresistenz, insbesondere der Koagulase-negativen Staphylokokken, ist die eingeschränkte Resistenzprüfung ein Problem, zumal nicht alle Antibiotika am Ort der Infektion gleich wirksam sind.

Bei der zu erwartenden Steigerung der Sensitivität der Methode durch die Nukleinsäure-Amplifikationstests besteht die Möglichkeit, dass bakterielle Kontaminationen, die bei Probengewinnung aus der Hautflora in das Untersuchungsmaterial gelangt sind, falsch positive Signale liefern können.

Diese Tests stellen eine Bereicherung in schwierigen Situationen dar und haben bezogen auf die Zukunft ein sehr großes Potenzial. Es werden allerdings Studien benötigt, die es ermöglichen, die Grenzen der Methode abzuschätzen und das bislang ungelöste Problem der unzureichenden Detektion des Resistenzverhaltens der Erreger zu lösen.

Molekularbiologische Untersuchungen sind zzt. ergänzend zur klassischen Mikrobiologie und bei speziellen Erregern (z. B. Mykobakterien, Borrelien, Streptokokken nach Antibiotikaexposition) sinnvoll.

Offene Fragen:

- Abgrenzung von Kontaminationen

- Resistenzprüfung der Erreger

\section{Schlussfolgerungen für die klinische Praxis}

Für die klinische Routine sollte im Rahmen einer Stufendiagnostik mindestens die Gelenkpunktion gefordert werden, die ggf. durch präoperative Biopsien ergänzt werden sollte. Die Ergebnisse sollten unter Einbeziehung von anamnestischen Daten, klinischen Untersuchungsergebnissen, klinisch-chemischen Befunden interdisziplinär bewertet werden. Bei Zweifeln an der Validität des Befundergebnisses sollte mindestens die Punktion wiederholt werden, besser die Gewinnung mehrerer Biopsien angestrebt werden.

In diesen Situationen und bei Suche nach ungewöhnlichen Erregern ergibt sich für die molekularbiologischen NukleinsäureAmplifikationstests eine Indikation.

Eine streng aseptische Gewinnung der Proben ist sowohl für die klassische mikrobiologische Diagnostik als auch für die molekularbiologische Diagnostik zu fordern, um Kontaminationen durch Bakterien der Hautflora weitestgehend auszuschließen.

Diese Befunde sollten durch mehrere intraoperative Biopsien ergänzt werden, um zu einem validen Erregernachweis zu gelangen, anhand dessen die begleitende Antibiotikatherapie korrigiert werden kann.

Stehen die Implantate zur Diagnostik zur Verfügung, ist die Sonikation anzustreben, die in der Lage ist, Erreger als Infektionsverursacher besser $\mathrm{zu}$ erkennen. Leider ist diese Methode zzt. nicht in ausreichendem Maße verfügbar.

Unabhängig von der verwendeten Technik ist eine strukturierte Kommunikation zwischen klinischen Anwendern und den im Labor tätigen erforderlich. 
Die Evaluierung der gefundenen Ergebnisse bedarf häufig des interdisziplinären Dialogs, um eine optimierte Therapie zu ermöglichen.

\section{Fazit für die Praxis}

Fremdkörperassoziierte Infektionen wie die periprothetische Infektion gehören nach wie vor zu den schwierig zu behandelnden Infektionen. Aufgrund der weitgehenden Unwirksamkeit der Antibiotika auf sessile Bakterien im Biofilm und der Tatsache, dass sessile Bakterien aufgrund des Biofilms nicht durch die zelluläre Abwehr eliminiert werden können, ist diese Infektion die ungünstige Kombination einer fremdkörperassoziierten Infektion mit einer Osteomyelitis.

Aufgrund der verzögerten Vermehrung und z.T. auch veränderten Stoffwechseleigenschaften der Erreger ist die Anzucht häufig nur aus Optimalmedien und nach verlängerter Beobachtungszeit möglich. Der Erregernachweis ist aber erforderlich, da die Empfindlichkeit der Erreger nicht vorhersehbar ist.

Da die Erreger zum überwiegenden Teil der Hautflora entstammen, müssen sowohl bei Gewinnung der Probe als auch bei Verarbeitung im Labor bakterielle
Kontaminationen aus der Hautflora vermieden werden.

Die Zusammenarbeit mit einem auf diesem Gebiet erfahrenen und infektiologisch versierten klinischen Mikrobiologen oder Infektiologen und ggf. Molekularbiologen verbessert die Ergebnisse der Diagnostik, ermöglicht eine rationale Therapie und wendet ggf. Schaden vom Patienten ab.

\section{Literatur}

1 Atkins BL, Athanasou N, Deeks JJ et al. Prospetive evaluation of criteria for microbiological diagnosis of prosthetic-joint infection at revision arthroplasty. J Clin Microbiol 1998; 36: 2932-2939

2 Fink B, Makowiak C, Fuerst $M$ et al. The value of synovial biopsy, joint aspiration and C-reactive protein in the diagnosis of late periprosthetic infection of total knee replacement. J Bone Joint Surg [Br] 2008; 90: 874-878

3 Frommelt L. Gelenkpunktat und Erregernachweis bei periprothetischer Infektion. Orthopäde 2008; 37: 1027-1034

${ }^{4}$ Herrmann $M$, Becker $K$, von Eiff $C$ et al. Infektionen der Knochen und des Knorpels, Teil I: Untersuchungsgang und Nachweismethoden und Teil II: Therapieprinzipien und spezielle Fragestellungen. In: Mauch H, Podbielski A, Herrmann M, Hrsg. Mikrobiologisch-infektiologische Qualitätsstandards (MIQ), MIQ 18 und 19. München, Jena: Urban \& Fischer; 2004

${ }^{5}$ Kriegsmann J, Hopf T, Jacobs D et al. Molekularpathogenetische Diagnostik von Gelenkinfektionen. Orthopäde 2009; 36: 531-538
${ }^{6}$ Mackowiak PA, Jones SR, Smith JW. Diagnostic value of sinus-tract cultures in chronic osteomyelitis. JAMA 1978; 239: 2772-2775

7 Schäfer P, Fink B, Sandow D et al. Prolonged bacterial culture to identify late periprosthetic joint infection: a promising strategy. Clin Infect Dis 2008; 47: 1403-1409

8 Spangehl MJ, Masri BA, Oconnell JX et al. Prospective analysis of preoperative and intraoperative investigation for the diagnosis of infection at the sites of two hundred and two revision total hip arthroplasties. J Bone Joint Surg [Am] 1999; 81: 672-682

9 Trampuz A, Hanssen AD, Osmon DR et al. Syovial fluid leukocyte count and differential for the diagnosis of prosthetic knee infection. Am J Med 2004; 117: 556-562

10 Trampuz A, Piper KE, Jacobson MJ et al. Sonication of removed hip and knee prostheses for diagnosis of infection. N Engl J Med 2007; 357: 654-663

\section{Dr. med. Lars Frommelt}

Arzt für Mikrobiologie und Infektionsepidemiologie, Konsiliararzt und Krankenhaushygieniker

Institut für Infektiologie,

Klinische Mikrobiologie und

Krankenhaushygiene

ENDO-Klinik Hamburg GmbH

Holstenstraße 2

22767 Hamburg

lars.frommelt@t-online.de 